\title{
Study of glycerol diffusion in skin and myocardium ex vivo under the conditions of developing alloxan-induced diabetes
}

\author{
Daria K. Tuchina ${ }^{1 *}$, Alexey N. Bashkatov ${ }^{1,2}$, Alla B. Bucharskaya ${ }^{3}$, Elina A Genina ${ }^{1,2}$, \\ and Valery V. Tuchin ${ }^{1,2,4}$ \\ ${ }^{1}$ N.G. Chernyshevsky Saratov National Research State University, 83 Astrakhanskaya Str., Saratov 410012, Russia \\ ${ }^{2}$ National Research Tomsk State University, 36 Lenin Ave., Tomsk 634050, Russia \\ ${ }^{3}$ V.I. Razumovsky Saratov State Medical University, 112 Bolshaya Kazachya Str., Saratov 410012, Russia \\ ${ }^{4}$ Institute of Precision Mechanics and Control, Russian Academy of Sciences, 24 Rabochaya Str., Saratov 410028, \\ Russia \\ *e-mail: tuchinadk@mail.ru
}

\begin{abstract}
Diabetes mellitus is one of widespread diseases, the development of which impairs the functioning of organism vital organs. The study of the processes that occur in tissues under diabetic conditions facilitates the development of diabetes diagnostics and treatment methods, as well as the prognosis of its complications. The paper reports a comparative study of glycerol diffusion in skin and myocardium of rats ex vivo in the normal condition and the conditions of alloxan-induced diabetes. The glycerol diffusion coefficients in tissues were determined basing on the analysis of kinetics of collimated light transmittance through tissue samples immersed in 70\%-glycerol solution. As a result, it was shown that the glycerol diffusion rate in the tissues of rats with alloxaninduced diabetes is essentially decreased as compared to the control group. (C) 2017 Journal of Biomedical Photonics \& Engineering.

Keywords: Skin; Myocardium; Diffusion coefficient; Permeability coefficient; Glycerol; Alloxan-induced diabetes; Collimated transmittance; Optical clearing.

Paper \#3155 received 1 Feb 2017; revised manuscript received 1 Mar 2017; accepted for publication 6 Mar 2017; published online 4 Apr 2017. doi: 10.18287/JBPE17.03.020302. [Special Issue. Workshop "Biophotonics" of the XIII all-Russian Youth Samara conference-contest on optics and laser physics].
\end{abstract}

\section{References}

1. “The Top 10 Causes of Death,” Fact Sheet №310, World Health Organization (WHO), May 2014.

2. J. E. Shaw, R. A. Sicree, and P. Z. Zimmet, "Global estimates of the prevalence of diabetes for 2010 and 2030," Diabetes Research and Clinical Practice 87(1), 4-14 (2010).

3. "10 facts about diabetes," World Health Organization (WHO), April 2016.

4. F. Quondamatteo, "Skin and diabetes mellitus: what do we know?" Cell and Tissue Research 355(1), 1-21 (2013).

5. N. Vigneshwaran, G. Bijukumar, N. Karmakar, S. Anand, and A. Misra, "Autofluorescence characterization of advanced glycation end products of hemoglobin," Spectrochimica Acta Part A: Molecular and Biomolecular Spectroscopy 61(1-2), 163-170 (2005).

6. J.-Y. Tseng, A. A. Ghazaryan, W. Lo, Y.-F. Chen, V. Hovhannisyan, S.-J.Chen, H.-Y. Tan, and C.-Y. Dong, "Multiphoton spectral microscopy for imaging and quantification of tissue glycation," Biomedical Optics Express 2(2), 218-230 (2010).

7. J. Blackwell, K. M. Katika, L. Pilon, K. M. Dipple, S. R. Levin, and A. Nouvong, "In vivo time-resolved autofluorescence measurements to test for glycation of human skin," Journal of Biomedical Optics 13(1), 014004 (2008). 
8. E. Selvin, M. W. Steffes, H. Zhu, K. Matsushita, L. Wagenknecht, J. Pankow, J. Coresh, and F. L. Brancati, "Glycated Hemoglobin, Diabetes, and Cardiovascular Risk in Nondiabetic Adults," The New England Journal of Medicine 362(9), 800-811 (2010).

9. N. I. Dikht, A. B. Bucharskaya, G. S. Terentyuk, G. N. Maslyakova, O. V. Matveeva, N. A. Navolokin, N. G. Khlebtsov, and B. N. Khlebtsov, "Morphological study of the internal organs in rats with alloxan diabetes and transplanted liver tumor after intravenous injection of gold nanorods," Russian Open Medical Journal 3(3), 0301 (2014).

10. A. N. Bashkatov, E. A. Genina, and V. V. Tuchin, "Measurement of glucose diffusion coefficients in human tissues," Chap. 19 in Handbook of Optical Sensing of Glucose in Biological Fluids and Tissues, V. V. Tuchin (ed.), Taylor \& Francis Group LLC, CRC Press, 587-621 (2009).

11. D. Zhu, K. V. Larin, Q. Luo, and V. V. Tuchin, "Recent progress in tissue optical clearing," Laser \& Photonics Reviews 7(5), 732-757 (2013).

12. L. M. Shevtsova, O. S. Bykova, N. P. Fedorova, M. V. Grigor'eva, and N. N. Maksimiuk, "Myocardium morphologic reorganization in experimental diabetes," Vestnik of Novgorod State University 2(85), 146-150 (2015) [in Russian].

13. A. Rohilla and S. Ali, "Alloxan induced diabetes: Mechanisms and effects," International Journal of Research in Pharmaceutical and Biomedical Sciences 3(2), 819-823 (2012).

14. V. D. Genin, D. K. Tuchina, A. J. Sadeq, E. A. Genina, V. V. Tuchin, and A. N. Bashkatov, "Ex vivo investigation of glycerol diffusion in skin tissue," J. of Biomedical Photonics \& Eng 2(1), 010303 (2016).

15. X. Wen, Z. Mao, Z. Han, V. V. Tuchin, and D. Zhu, "In vivo skin optical clearing by glycerol solutions: mechanism," Journal of Biophotonics 3(1), 44-52 (2010).

16. T. Son, and B. Jung, "Cross-evaluation of optimal glycerol concentration to enhance optical tissue clearing efficacy," Skin Research and Technology 21(3), 327-232 (2015).

17. Physical properties of glycerine and its solutions, Glycerine Producers' Association, New York (1963).

18. D. K. Tuchina, R. Shi, A. N. Bashkatov, E. A. Genina, D. Zhu, Q. Luo, and V. V. Tuchin, "Ex vivo optical measurements of glucose diffusion kinetics in native and diabetic mouse skin," J. Biophotonics 8(4), 332-346 (2015).

19. A. Kotyk, and K. Janacek, Membrane Transport: An Interdisciplinary Approach, Plenum Press, NewYork (1977). ISBN 978-1-4684-3333-3.

20. A. N. Bashkatov, E. A. Genina, and V. V. Tuchin, "Optical properties of skin, subcutaneous, and muscle tissues: a review," Journal of Innovative Optical Health Sciences 4(1), 9-38 (2011).

21. S. Tanaka, G. Avigad, B. Brodsky, and E. F. Eikenberry, "Glycation induces expansion of the molecular packing of collagen,” Journal of Molecular Biology 203(2), 495-505 (1988).

22. B.-M. Kim, J. Eichler, K. M. Reiser, A. M. Rubenchik, and L. B. Da Silva, "Collagen structure and nonlinear susceptibility: effects of heat, glycation, and enzymatic cleavage on second harmonic signal intensity," Lasers in Surgery and Medicine 27(4), 329-335 (2000).

23. E. L. Hull, M. N. Ediger, A. N. T. Unione, E. K. Deemer, M. L. Stroman, and J. W. Baynes, "Noninvasive, optical detection of diabetes: model studies with porcine skin," Optics Express 12(19), 4496-4510 (2004).

24. H. N. Mayrovitz, A. McClymont, and N. Pandya, "Skin tissue water assessed via tissue dielectric constant measurements in persons with and without diabetes mellitus," Diabetes Technology \& Therapeutics 15(1), 6065 (2013).

25. O. S. Khalil, "Non-Invasive Glucose Measurement Technologies: An Update from 1999 to the Dawn of the New Millennium," Diabetes Technology \& Therapeutics 6(5), 660-697 (2004).

26. W. Hanna, D. Friesen, C. Bombardier, D. Gladman, and A. Hanna, "Pathologic features of diabetic thick skin,” J. Am. Acad. Dermatol. 16(3), 546-553 (1987).

27. D. K. Tuchina, A. N. Bashkatov, E. A. Genina, and V. V. Tuchin, "Quantification of glucose and glycerol diffusion in myocardium," J. Innovative Optical Health Science 8(3), 1541006 (2015).

28. E. A. Genina, A. N. Bashkatov, M. D. Kozintseva, and V. V. Tuchin, "OCT study of optical clearing of muscle tissue in vitro with 40\% glucose solution," Optics and spectroscopy 120(1), 20-27 (2016).

29. L. M. Oliveira, M. I. Carvalho, E. M. Nogueira, and V. V. Tuchin, "Diffusion characteristics of ethylene glycol in skeletal muscle," Journal of Biomedical Optics 20(5), 051019 (2014).

30. X. Guo, Z. Guo, H. Wei, H. Yang, Y. He, S. Xie, G. Wu, X. Deng, Q. Zhao, and L. Li, "In vivo comparison of the optical clearing efficacy of optical clearing agents in human skin by quantifying permeability using optical coherence tomography," Photochemistry and Photobiology 87(3), 734-740 (2011).

31. H. Schaefer, and T. E. Redelmeier, Skin Barrier: Principles of percutaneous absorption, Karger, Basel (1996). ISBN: 9783805563260

32. O. S. Zhernovaya, V. V. Tuchin, and I. V. Meglinski, "Monitoring of blood proteins glycation by refractive index and spectral measurements," Laser Physics Letters 5(6), 460-464 (2008).

33. G. Mazarevica, T. Freivalds, and A. Jurka, "Properties of erythrocyte light refraction in diabetic patients," J. Biomed. Opt. 7(2), 244-247 (2002). 
34. E. M. Culav, C. H. Clark, and M. J. Merrilees, "Connective tissue: matrix composition and its relevance to physical therapy,” Phys. Therapy 79, 308-319 (1999).

35. R. K. Murray, D. K. Granner, P. A. Mayes, and V. W. Rodwell, Harper's biochemistry, Appleton \& Lange, Norwalk, California (1988). ISBN: 9780838535622

36. C. Molteni, and M. Parrinello, "Glucose in aqueous solution by first principles molecular dynamics," Journal of the American Chemical Society 120(9), 2168-2171 (1998).

37. J. W. Wiechers, J. C. Dederen, and A. V. Rawlings (eds.), Skin Moisturization, Taylor \& Francis Inc. (2009).

38. D. K. Tuchina, Study of Diffusion of Chemical Agents in Biological Tissues Using Optical Methods in the Normal Conditions and in the Presence of Model Diabetes. Diss. Cand. Sci. in Phys. and Math., Saratov National Research State University, Saratov, Russia (2016) [in Russian].

\section{Introduction}

Diabetes mellitus is an endocrine disease characterised by the increased content of glucose in the organism. According to the data of the World Health Organisation in 2012 - 2013 the diabetes mellitus caused nearly five millions deaths, which made it the eighth factor of mortality in the world [1]. The worldwide number of patients suffering from diabetes mellitus in 2030 is expected to increase by $54 \%$ as compared to 2010 [2], thus moving diabetes to the seventh position in the list of dangerous diseases [3]. One-third part of all patients with diabetes have complications in the form of skin lesions, the chronic hardly healed ulcers being the severest of them, fraught with the risk of infecting the entire organism [4]. The complications of diabetes mellitus are mainly related to the glycation of the human organism proteins, which comprise a considerable part of many tissues. Protein glycation is initiated by the non-enzymatic reaction between the protein amides and the sugar carbonyl group followed by the cross-linking of proteins $[5,6]$. The glycation of proteins modifies the structure of tissues, restricts their functioning [6-8], causes metabolic imbalance and, finally, impairs the organ functioning [5, 9].

Since the permeability of tissue for chemical agents is determined by its structure [10], the variation of the diffusion behaviour and rate in the tissue reflects the structure change, which can be used as a biomarker [11], e.g., a biomarker of the tissue protein glycation degree. In turn, the degree of tissue glycation in available organs, such as skin, mucous and submucous membranes of hollow organs can be used to diagnose the tissue condition in hardly accessible organs, such as myocardium, retina, brain, thus predict the vitally dangerous complication of diabetes.

To quantify the diffusion rate of an agent in the tissue, one can use the method of tissue optical clearing [10] based on the action of a biocompatible hyperosmotic chemical agent (immersion agent) on the tissue. It is well known that tissues possess sufficiently strong light scattering due to the inhomogeneity of refractive index of their components. The action of the immersion agent on the tissue causes partial replacement of interstitial fluid by the agent and temporal dehydration of the tissue, which leads to refractive index matching between the tissue components and temporary tissue packing. Finally, both processes reduce the light scattering in the tissue. The rate of light scattering reduction in the tissue is determined by the rate of the agent diffusion.

The present paper is devoted to the comparative study of glycerol diffusion rate in skin and myocardium of rats in normal condition and under the conditions of model alloxan-induced diabetes, the aim of the study is to determine the relation between the optical and diffusion properties of skin and myocardium in the course of model diabetes development.

\section{Materials and methods}

The studies were carried out using ex vivo samples of skin and myocardium of white outbred male laboratory rats having the weight of $500 \pm 20 \mathrm{~g}$. Hair from the skin surface was preliminarily removed by depilatory cream «Veet» (Reckitt Benckiser, France). The skin was treated with saline to remove the residual cream from the surface of the samples. The skin samples were extracted using surgical scissors, and then the subcutaneous fat hampering the penetration of hydrophilic agents into the dermis was removed from them. The samples of myocardium in vitro were prepared using a scalpel after the preliminary freezing of the whole heart of a rat. Before the measurements, the myocardium samples were washed in saline to remove the blood excess from the samples. In each series of experiments, ten samples were used, each of them having the area of nearly $10 \times 15 \mathrm{~mm}^{2}$.

The laboratory animals were divided into the control group and the diabetic one. The development of model diabetes in the rats of the diabetic group was induced by a single intramuscular injection of alloxan (Acros Organic, Belgium) [4, 9, 12, 13], mixed with saline in the proportion of $10 \mathrm{mg}$ of alloxan per $100 \mathrm{~g}$ of the rat body mass. The mean values of free glucose in the blood taken after fasting from the caudal vein were measured by glucometer "Accu-Chek Performa" (Roche Diagnostics, Germany). Before alloxan injection, on the 7-th day after injection and on the day of experiment (on the 15-th day after the injection) the free glucose concentration amounted to $128 \pm 18,411 \pm 131$, and $350 \pm 147 \mathrm{mg} / \mathrm{dl}$, respectively. Thus, glucose level in blood increased by nearly 3 times, which is characteristically for diabetes. No alloxan was injected to the rats of the control group. 
The experimental protocol on animal research was compiled as per the Helsinki Declaration, 2000 and approved by V.I. Razumovsky Saratov State Medical University ethical committee.

The aqueous $70 \%$-solution of glycerol, used as an immersion agent, was prepared by mixing the dehydrated glycerol ("Trading depot No.1 of Chemicals", Russia) with the distilled water. The refractive index of the solution $n=1.427 \pm 0.001$ was measured at the wavelength $589 \mathrm{~nm}$ by the Abbe refractometer IRF-454B2M (LOMO, Russia).
Glycerol solutions of different concentrations are frequently used for optical clearing of tissues [11, 1416]. Our choice of concentration for the glycerol solution was determined by its relatively low viscosity $(22.5 \mathrm{cP})$ in comparison with the dehydrated glycerol $(1410.0 \mathrm{cP})$ [17] and yet sufficiently high refractive index of 1.427 [17] (Table 1). Moreover, the studies of the efficiency of skin optical clearing using aqueous glycerol solutions of different concentrations [11, 14, $16]$ have shown that the concentration of $70 \%$ is close to optimal.

Table 1 Refractive index and viscosity of glycerol solutions of different concentration [17].

\begin{tabular}{cccccccc}
\hline Concentration of glycerol solution, \% & 40 & 50 & 60 & 70 & 80 & 90 & 100 \\
\hline Refractive index & 1.384 & 1.398 & 1.413 & 1.427 & 1.443 & 1.458 & 1.474 \\
\hline Viscosity, cP & 3.7 & 6.0 & 10.8 & 22.5 & 50.1 & 219.0 & 1410.0 \\
\hline
\end{tabular}

The spectra of collimated transmittance of the tissue samples were measured using the multichannel spectrometer USB4000-Vis-NIR (Ocean Optics, USA). The tissue sample was fixed at the plastic plate having the area $3.5 \times 1.5 \mathrm{~cm}^{2}$ with a hole in the centre having the area $8 \times 8 \mathrm{~mm}^{2}$ and placed in the glass cuvette having the volume $5 \mathrm{ml}$ filled with the glycerol solution. The cuvette was installed between two fibre-optical cables QP400-1-VIS-NIR (Ocean Optics, USA) with the inner diameter $400 \mu \mathrm{m}$. To provide the beam collimation, the collimators 74-ACR (Ocean Optics, USA) were fixed at the ends of fibres by the standard connectors SMA-905. The halogen lamp HL-2000 (Ocean Optics, USA) was used as a source of light. The setup is schematically shown in Fig. 1. All measurements were performed at room temperature $\left(\sim 20^{\circ} \mathrm{C}\right)$.

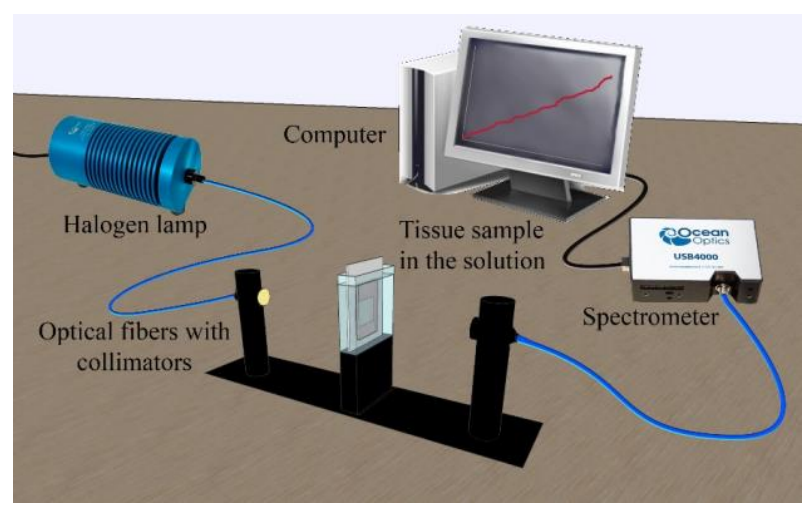

Fig. 1 Schematic diagram of the experimental setup for the measurement of collimated transmittance of ex vivo tissue samples.

The thickness and weight of the samples were measured before and after their placing into glycerol solution. For the thickness measurement, the samples were placed between two microscope slides. The measurements were performed at five points using a micrometer with the accuracy of $\pm 5 \mu \mathrm{m}$ and then averaged. The weight of the samples was measured using the electronic balance (Scientech, USA) with the accuracy of $\pm 1 \mathrm{mg}$.

The measured kinetics of collimated transmittance of tissue samples were used for calculating the efficiency of their optical clearing, the coefficients of glycerol diffusion in tissues, and the permeability coefficients of biological tissues for glycerol. The method of estimating the diffusion coefficients for chemical agents used in the present study is described in detail in Refs. [10, 18]. In the calculations we allowed for the fact that glycerol diffuses into the skin mainly from the dermis side, while in the case of myocardium glycerol penetrates into the sample from both sides of the sample. To estimate the skin permeability coefficient $P$ for glycerol (the case of one-sided diffusion), we used the expression [19]:

$$
P=\frac{D}{l},
$$

where $D$ is the glycerol diffusion coefficient in the tissue, $l$ is the tissue sample thickness.

The permeability coefficient of myocardium (the case of two-sided diffusion) was calculated using the following expression [19]:

$$
P=\frac{\pi^{2} D}{l} .
$$

The optical clearing efficiency for a tissue sample was determined at different wavelengths as the relative change of the scattering coefficient of the tissue under the action of the immersion agent:

$$
\mathrm{OC}_{e f f}=\frac{\mu_{S 0}-\mu_{S \min }}{\mu_{s 0}},
$$

where $\mu_{\mathrm{s} 0}$ and $\mu_{\mathrm{smin}}$ are the initial and the minimal value of the scattering coefficient of the tissue sample determined from the values of attenuation coefficient $\left(\mu_{t}=\mu_{a}+\mu_{s}\right)$. The latter was calculated from the 
values of collimated transmittance using the BouguerLambert law, $\mu_{t}=-\ln \left(T_{c} / l\right)$, where $T_{\mathrm{c}}$ is the collimated transmittance of the tissue sample, $l$ is the sample thickness, $\mu_{\mathrm{a}}$ is the absorption coefficient of the tissue. In the calculations, we used the values of the absorption coefficient presented in Ref. [20]. Since for both skin and muscle tissue, the absorption coefficient is much smaller than the scattering coefficient [20], we did not consider the possible change of the absorption coefficient due to the osmotic dehydration of the tissue in our calculations.

\section{Results and discussion}

Figure 2 presents the images of skin $(\mathrm{a}, \mathrm{b})$ and myocardium (c, d) samples before $(a, c)$ and after $(b, d)$ the optical clearing in $70 \%$-glycerol solution. The figure clearly demonstrates the effect of optical clearing of the studied tissues, i.e., the increase of light transmission in the visible range.

Figure 3 presents the typical spectra $(a, b)$ and the kinetics of collimated transmittance (c, d) of rat skin samples of the control (a, c) and diabetic (b, d) groups during the optical clearing in the $70 \%$-glycerol solution. In both cases, it is well seen that the collimated

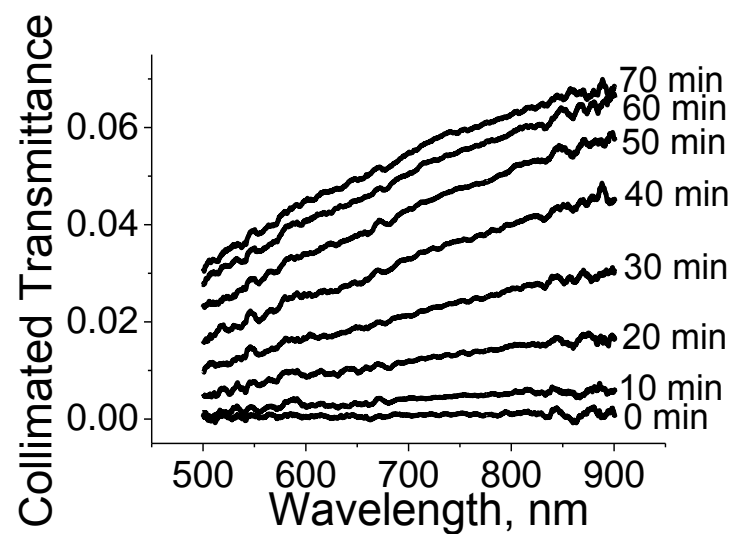

a

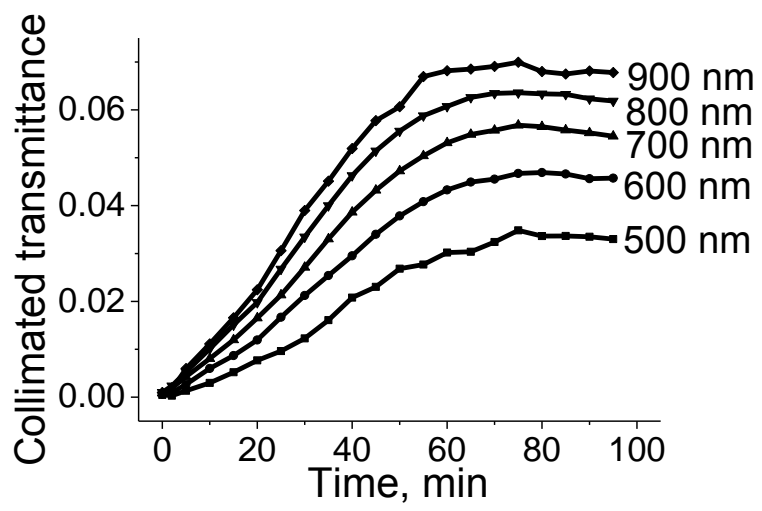

c transmittance increases and reaches saturation. However, from the comparison of the presented plots it can be seen that it takes more time (approximately 100 minutes) for the diabetic rat skin sample, as compared with that from the control group (nearly 70 minutes) to achieve nearly the same level of optical transmittance.
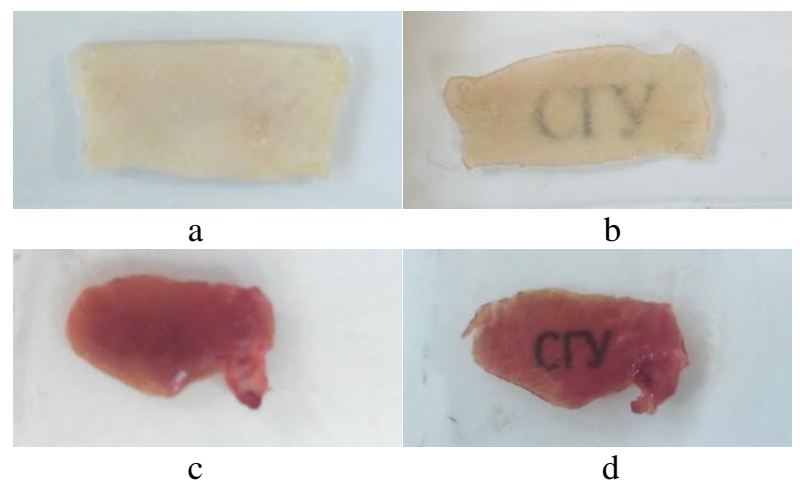

Fig. 2 Images of skin (a, b) and myocardium (c, d) samples before $(a, c)$ and after $(b, d)$ the optical clearing in $70 \%$-glycerol solution.

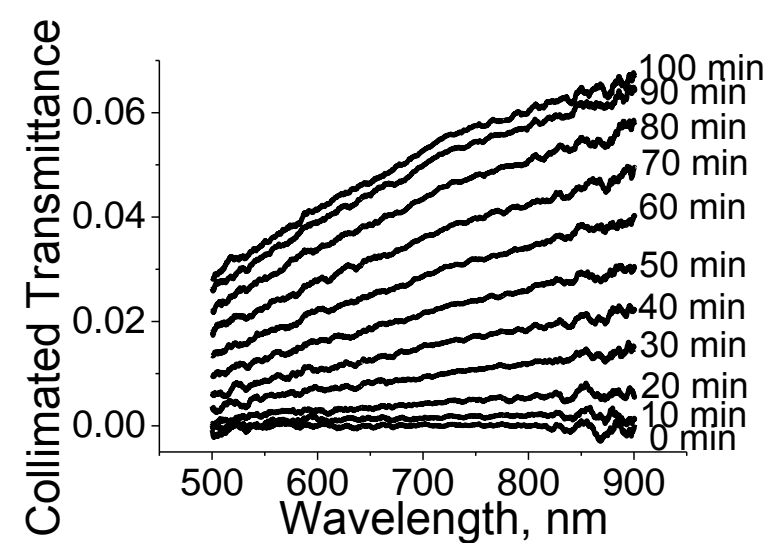

b

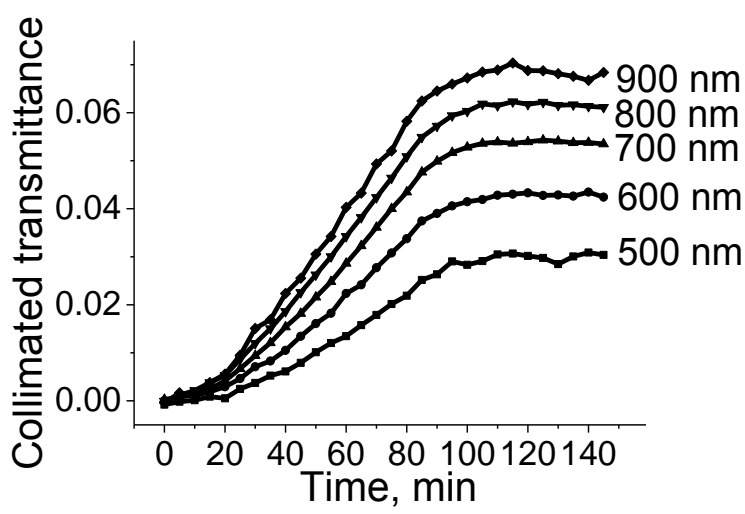

d

Fig. 3 Spectra (a, b) and kinetics (c, d) of collimated transmittance for the rat skin samples from the control (a, c) and diabetic (b, d) groups during the optical clearing with $70 \%$-solution of glycerol. 
Figure 4 presents the typical time dependences of the collimated transmittance of rat myocardium samples from the control and diabetic groups in the course of optical clearing. Similar to the case of skin (Fig. 3), in both cases we observed the growth of collimated transmittance, and in the control group the optical clearing of myocardium samples achieves the saturation faster (approximately in 20 minutes of immersion) than in the diabetic group (more than 30 minutes). At the

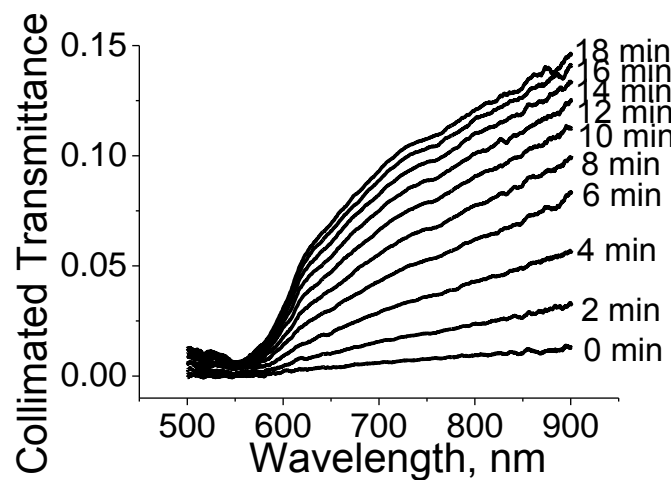

a

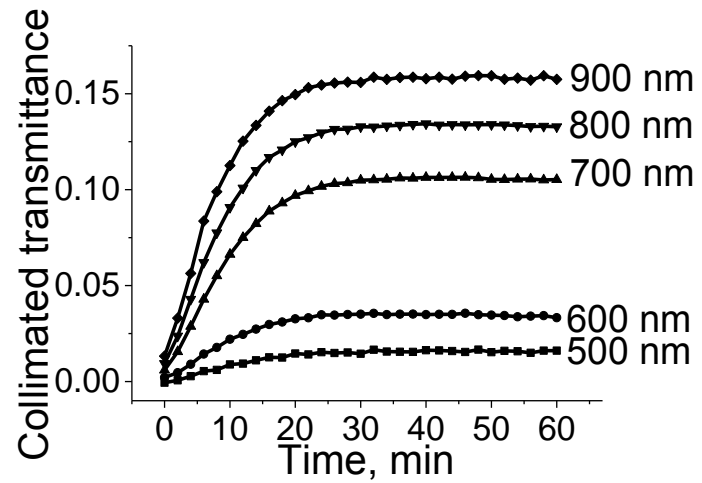

$\mathrm{c}$ appropriate wavelengths, the transmittance reaches nearly the same final values in both control and diabetic groups. In the wavelength range 500-600 $\mathrm{nm}$ the transmittance has relatively low values, which is due to the light absorption by myoglobin. However, in this range the growth of transmittance due to the optical clearing, i.e., the reduction of light scattering, is also observed.

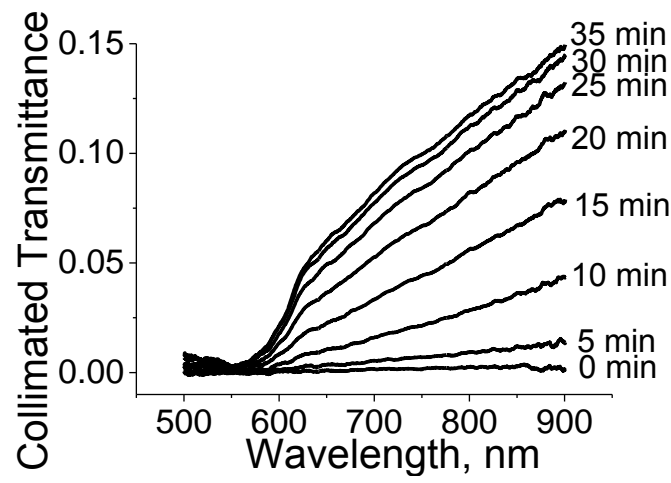

b

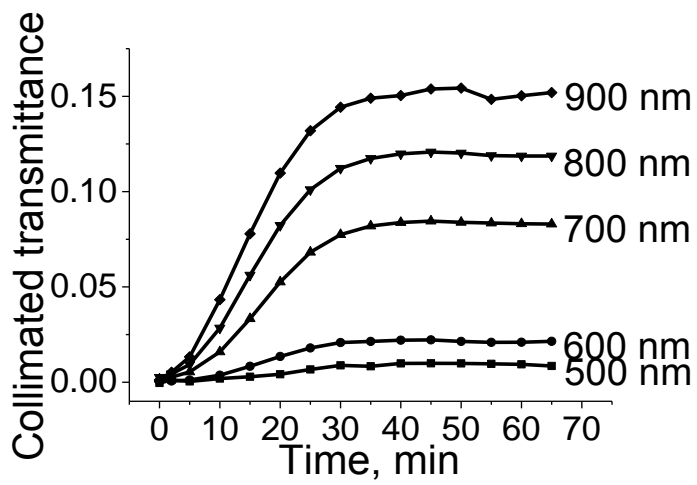

d

Fig. 4 Spectra (a, b) and kinetics (c, d) of collimated transmittance of rat myocardium samples from the control (a, c) and the diabetic $(b, d)$ groups during the optical clearing with $70 \%$-glycerol solution.

Table 2 summarises the mean values of thickness and weight of the tissue samples before $\left(l_{0}\right.$ and $\left.W_{0}\right)$ and after $(l$ and $W)$ the optical clearing, the glycerol diffusion coefficients $(D)$, and the permeability $(P)$ of the tissue samples for glycerol, as well as the optical clearing efficiency in the tissue samples $\left(\mathrm{OC}_{\mathrm{eff}}\right)$ at different wavelengths for the control and the diabetic groups. The reduction of weight (see Table 2) of skin and myocardium samples and thickness (for myocardium) is a result of glycerol-induced sample dehydration, contributing the optical clearing. It is interesting to note that the loss of weight is more expressed in the tissue samples from the control group of rats. The loss amounts to nearly $29 \%$ of the initial mass for both the skin and the myocardium in the control group, while in the diabetic group the weight loss is $\sim 24 \%$ for the skin and $\sim 26 \%$ for the myocardium.
The insignificant thickness increase of the skin samples lies within the measurement error and was neglected. However, the obviously different behaviour of the thickness of skin and myocardium samples indicates the difference of structural organisation of these tissues and, correspondingly, the difference in their response to the osmotic action of glycerol. As compared to myocardium, the skin reacts on the transverse compression much slower and weaker, and hence the osmotic effect in skin manifests itself in stronger longitudinal compression, which leads to insignificant (within the measurement error) increase of the sample thickness. The used method of measuring the collimated transmission with relatively small diameters of the light beam in the samples allows one to neglect the sample area variation (the longitudinal compression) in the algorithm calculating the diffusion coefficient. 
Table 2 Mean values of the thickness and weight of tissue samples before $\left(l_{0}\right.$ and $\left.W_{0}\right)$ and after $(l$ and $W)$ optical clearing, the glycerol diffusion coefficient $(D)$, the tissue permeability $(P)$, and the efficiency of optical clearing $\left(\mathrm{OC}_{\mathrm{eff}}\right)$ at different wavelengths for the skin and myocardium samples of rats from the control (c) and diabetic (d) groups.

\begin{tabular}{|c|c|c|c|c|c|c|c|c|}
\hline Tissue & $\mathrm{c} / \mathrm{d}$ & $l_{0} / l, \mathrm{~mm}$ & $W_{0} / W, \mathrm{mg}$ & $D, \mathrm{~cm}^{2} / \mathrm{s}$ & $\mathrm{P}, \mathrm{cm} / \mathrm{s}$ & $\begin{array}{c}\mathrm{OC}_{\mathrm{eff}} \\
(600 \mathrm{~nm})\end{array}$ & $\begin{array}{c}\mathrm{OC}_{\mathrm{eff}} \\
(700 \mathrm{~nm})\end{array}$ & $\begin{array}{c}\mathrm{OC}_{\text {eff }} \\
(800 \mathrm{~nm})\end{array}$ \\
\hline \multirow{2}{*}{$\frac{\pi}{\sqrt[n]{n}}$} & $\mathrm{c}$ & $\begin{array}{c}0.53 \pm 0.11 / \\
0.55 \pm 0.13\end{array}$ & $\begin{array}{c}271 \pm 68 / \\
183 \pm 21\end{array}$ & $(8.33 \pm 2.60) \times 10^{-7}$ & $(1.68 \pm 0.88) \times 10^{-5}$ & $0.58 \pm 0.09$ & $0.56 \pm 0.04$ & $0.57 \pm 0.05$ \\
\hline & $\mathrm{d}$ & $\begin{array}{c}0.56 \pm 0.04 / \\
0.57 \pm 0.07\end{array}$ & $\begin{array}{c}270 \pm 32 / \\
203 \pm 73\end{array}$ & $(6.77 \pm 2.11) \times 10^{-7}$ & $(1.20 \pm 0.33) \times 10^{-5}$ & $0.60 \pm 0.07$ & $0.64 \pm 0.04$ & $0.65 \pm 0.06$ \\
\hline \multirow{2}{*}{ 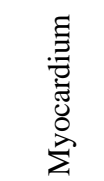 } & $\mathrm{c}$ & $\begin{array}{c}0.68 \pm 0.11 / \\
0.51 \pm 0.09\end{array}$ & $\begin{array}{c}210 \pm 37 / \\
146 \pm 35\end{array}$ & $(7.90 \pm 3.61) \times 10^{-7}$ & $(11.8 \pm 6.1) \times 10^{-5}$ & $0.51 \pm 0.07$ & $0.59 \pm 0.06$ & $0.61 \pm 0.07$ \\
\hline & $\mathrm{d}$ & $\begin{array}{l}0.58 \pm 0.06 / \\
0.47 \pm 0.07\end{array}$ & $\begin{array}{c}214 \pm 41 / \\
153 \pm 36\end{array}$ & $(5.14 \pm 2.10) \times 10^{-7}$ & $(8.60 \pm 3.21) \times 10^{-5}$ & $0.53 \pm 0.02$ & $0.64 \pm 0.05$ & $0.66 \pm 0.06$ \\
\hline
\end{tabular}

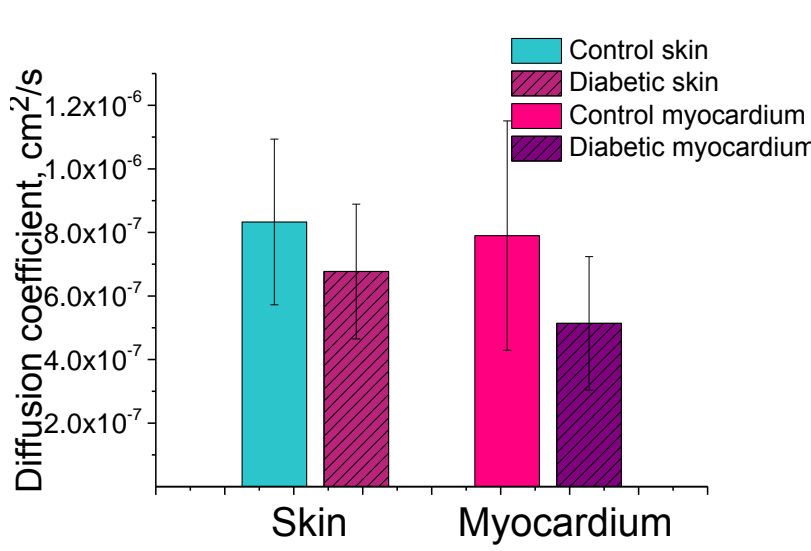

a

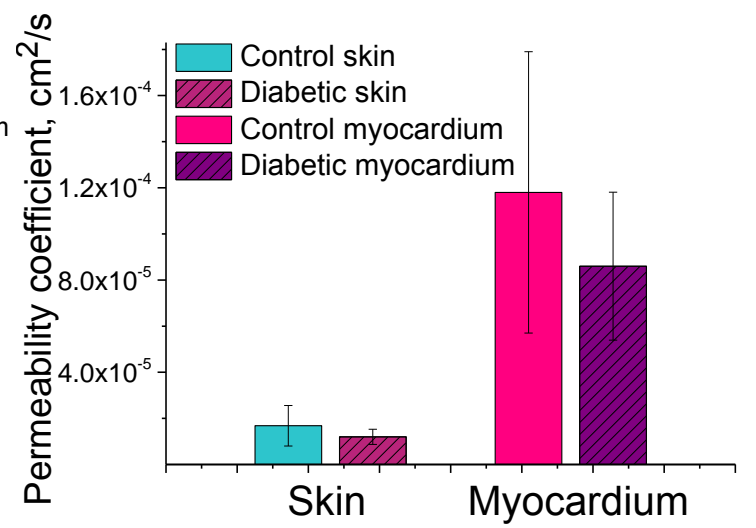

b

Fig. 5 Glycerol diffusion coefficients in skin and myocardium (a) and permeability coefficients (b) of the studied tissues from diabetic and control groups.

Figure 5 presents the histogram of glycerol diffusion coefficient in the skin and myocardium of rats from the control and the diabetic groups. It is clearly seen that in rats with the model alloxan-induced diabetes the measured diffusion and permeability coefficients take smaller values.

The reduction of the glycerol diffusion rate in the tissue under the conditions of developing diabetes may be related to its structural modification, including the degree of fibril packing, the cross-linking of proteins, and the change of free and bound water content in the skin [6-8, 21-23]. Obviously, the change of free and bound water balance reflects the change in the structure of fibrils and interfibrilar space and, correspondingly, the diffusion rate for the clearing agent molecules in the tissue. The authors of Ref. [22] demonstrated the increase of the scattered light intensity and, therefore, the increase of diffuse reflectance in the glycated tissue, and the authors of Refs. $[12,24]$ reported the increase of water content in the tissue, which allows to judge on the processes of the relevant tissue modifications during the diabetes development. Different reflectance of light by intact and diabetic skin, caused by glycation of tissue proteins, is discussed in the review paper [25]. The change of the tissue optical properties is a consequence of its structural modification. It was shown that the glycation leads to the loss of axial packing of the collagen-I fibrils due to the twisting and distortion of the matrix by the glycation adducts [21-23, 26]. The increased water content in skin, found in the patients with diabetes mellitus [24], the intercellular and intermuscular edema [12] can also cause increased scattering of light in the tissue.

Thus, the impairment of the tissue permeability (due to the formation of interfibril links) should be related to the impairment of metabolism, which can, probably, lead to the development of larger-scale complications in a diabetic patient.

In Refs. [9, 12] the effect of developing alloxaninduced diabetes on the internals of laboratory animals was studied. The authors of Ref. [9] report the morphologic changes of different degree of severity in the tissues of liver, kidneys, spleen, and pancreas in rats on the 15-th day after the alloxan injection as compared 
to the control group of animals. In particular, they observed the decreased accumulation of glycogen in liver, the necrosis of individual cells in kidneys, and in the pancreas, the fibrous perivascular indurations were found and the size and the number of pancreatic islands was reduced.

The structural changes of myocardial tissues in laboratory rats under the model diabetes were studied in Ref. [12]. During the first three weeks after the alloxan injection the morphological manifestations of the myocardium restructuring under the development of alloxan-induced diabetes were revealed. As a result, the authors observed dissociation, destruction, hypertrophy, fragmentation of cardiomyocytes, thinning, twisting, and destructive changes of muscular fibrils of the myocardium. The increased number of collagen fibres by 2.8 times as compared to the intact group of animals and the accumulation of protein in the muscle cells were also found, which lead to the increased viscosity of sarcoplasm, hardness and strength of myofilaments, subject to the increased mechanical load.

Thus, the data of Refs. [9, 12, 21-23, 26] confirm our conclusion that in the glycated tissues the deceleration of glycerol diffusion process takes place. The value of the glycerol diffusion coefficient in the rat myocardium, obtained in the present work for the control group $\left((7.90 \pm 3.61) \times 10^{-7} \mathrm{~cm}^{2} / \mathrm{s}\right)$ is close to the glycerol diffusion coefficient in porcine myocardium $\left((7.71 \pm 4.63) \times 10^{-7} \mathrm{~cm}^{2} / \mathrm{s}\right)$, measured earlier in Ref. [27]. The glucose diffusion coefficient in the bovine abdominal muscle tissue, measured in vitro using OCT, appeared to be $(2.98 \pm 0.94) \times 10^{-6} \mathrm{~cm}^{2} / \mathrm{s}$, the sample thickness in this case being increased [28] due to the $\mathrm{pH}$ difference of the used solutions. In the study of optical clearing of rat muscle tissue using $60 \%$-ethylene glycol solution, the water diffusion coefficient $D_{\text {water }}=3.12 \times 10^{-6}$ $\mathrm{cm}^{2} / \mathrm{s}$ was found as for flow induced by the hyperosmolarity of ethylene glycol solution [29]. When using the aqueous 54\%-glucose solution as an immersion agent, the diffusion coefficient of the induced water flow in the muscle tissue had the close value, $D_{\text {water }}=3.22 \times 10^{-6} \mathrm{~cm}^{2} / \mathrm{s} \quad$ [29]. Thus, the dehydration seems to be faster than the glycerol diffusion in skin and myocardium. The discussed difference in the diffusion rate of agents in the muscle tissue is caused by the difference in the physical properties of agents, the structure of the studied tissues, and the methods of measuring the diffusion rate of the agent.

The skin permeability for glycerol (at using 70\%glycerol solution) measured in the present paper $\left((1.68 \pm 0.88) \times 10^{-5} \mathrm{~cm} / \mathrm{s}\right)$, is higher by an order of magnitude obtained in the studies of human skin in vivo, $(1.67 \pm 0.04) \times 10^{-6} \mathrm{~cm} / \mathrm{s}$ [30], with the $40 \%$-glycerol solution. The reason of higher permeability observed in the present work is the greater (almost by two times) osmolarity of more concentrated glycerol solution, which means that free water becomes to be involved in the total flow in the tissue. Water has much higher diffusion rate than glycerol [14]. The experimental conditions (in vivo and ex vivo) and the methods of optical measurements (collimated transmittance and OCT) also contribute to these differences. Obviously, when the solution is applied to the skin surface in vivo, the glycerol penetrates into the skin from the epidermis side, which inhibits the diffusion of glycerol into skin due to its protective properties [31]. On the contrary, in the present work the action of the solution on the skin occurs mainly from the dermis side, which facilitates the process of its optical clearing.

Table 2 also presents the efficiency of optical clearing of tissue samples at different wavelengths using the $70 \%$-glycerol solution. It can be seen that the efficiency of optical clearing is higher in diabetic tissues than in control ones, which is caused by smaller initial optical transmittance of the diabetic tissue determined by the increased light scattering [18]. The fact that diabetic tissue scatters light stronger than a normal one can be explained by three reasons: i) the increased number and irregular packing of collagen fibres in myocardium [12], i.e., the increased scattering cross section and number of scatterers; ii) the increased refractive index of collagen and other proteins due to the glycation [32, 33]; iii) the increased level of free glucose, holding the water in the interfibrilar space. The last reason is due to the fact that in the presence of diabetes the formation of hydrated shell of proteins is implemented not only via the interaction of water molecules with glycosaminoglycans of the interstitial matrix [34, 35], but also via the interaction of water with glucose molecules, each of them known to bind up to ten water molecules [36]. All these factors lead to the additional tissue hydration and, correspondingly, to the reduction of the refractive index of the interstitial fluid, which increases light scattering. The efficiency of optical clearing is higher because the values of the minimal scattering coefficient $\mu_{\text {smin }}$ achieved in the skin of rats from both control and diabetic groups are close, while the initial scattering coefficient $\mu_{\mathrm{s} 0}$ is higher for the skin of rats from the control group (see Eq. (3)). Physically it means that because of hindered diffusion of the agent and water molecules in diabetic samples the process is slower, the volume of the impregnated tissue increases, and the process of clearing is finished later, when the balance of all flows is established, but with nearly the same resulting tissue transparency in the both control and diabetic groups.

The influence of glucose molecules also explains the smaller loss of weight in diabetic tissues (see Table 2), since the glucose in these tissues possesses higher hygroscopicity as compared to the glycerol, each molecule of which binds only six molecules of water [37]. Thus, the glucose contained in the diabetic tissues inhibits the osmotic dehydration of tissues caused by glycerol.

It is well seen from Table 2 that for the tissues of the diabetic group the efficiency of optical clearing increases with the growth of the wavelength $\lambda$ : slightly for skin, from $0.60 \pm 0.07(\lambda=600 \mathrm{~nm})$ to $0.65 \pm 0.06$ $(\lambda=800 \mathrm{~nm})$, and essentially greater for myocardium, 
from $0.53 \pm 0.02(\lambda=600 \mathrm{~nm})$ to $0.66 \pm 0.06(\lambda=800 \mathrm{~nm})$. For control sample of myocardium, the efficiency of optical clearing also increases with the growth of wavelength, but the effect is expressed weaker than in the diabetic tissue. At the same time, optical clearing efficiency of control skin does not depend on the wavelength. Generally, such behaviour can be explained by the fact that the difference between the refractive indices of the scatterers and the interstitial fluid, saturated with glycerol, decreases with the growth of the wavelength [38], leading to better matching of refractive indices, the reduction of light scattering, and the increase of the tissue optical clearing efficiency. Somewhat greater increase of optical clearing efficiency with the growth of the wavelength in diabetic tissues can be, probably, related to the behaviour of dispersion dependences of the refractive index of tissue scatterers, caused by the difference of their hydration degree.

\section{Conclusion}

In the reported studies, we have found that the optical clearing of rat skin and myocardium is slower under the conditions of alloxan-induced diabetes. The glycerol diffusion rate in skin and myocardium and the permeability of diabetic tissues for glycerol are smaller as compared to the control group. The obtained values of glycerol diffusion coefficient in skin and myocardium of the rats from control group are higher than in the diabetic group by 1.23 and 1.54 times, respectively. The permeability of skin and myocardium in the control group of rats is higher, than for the model diabetes by 1.4 and 1.3 times, respectively. However, the optical clearing efficiency of diabetic tissues is higher, than that of healthy ones.

Thus, it is established that the diffusion of glycerol molecules in the tissues of rat myocardium and skin is hampered by the development of alloxan-induced diabetes. This result offers a possibility of developing a method for early diagnostics of diabetes mellitus complications, in particular, for assessing the degree of myocardial lesion evidence by the stage of skin glycation in the process of diabetes development and treatment. It is experimentally proved that for both studied tissues of the diabetic group the increase of the optical clearing efficiency with the growth of the wavelength is considerably higher than in the control group.

\section{Acknowledgments}

The studies were carried out under the support from the Russian Scientific Foundation, grant No. 14-15-00186 (the diabetes model, the technique of collimated transmission measurement, the algorithm for diffusion coefficient determination), from the President of Russian Federation, grant for the State Support of Leading Scientific Schools NSh-7898.2016.2 (the model of optical clearing in the presence of alloxaninduced diabetes), and the programme U.M.N.I.K., agreement No. 9084GU2/2015 (Tuchina D.K.) (the comparative study of diffusion rate in skin and myocardium). 